\title{
X-80, A EUROPEAN X-RAY ASTROPHYSICS MISSION
}

\author{
B. G. Taylor', R. Berthelsdorf 2 , A. C. Brinkman ${ }^{3}$, \\ J. Lemen ${ }^{2}$, N. Lund 4 , H. Olthof ${ }^{5}$, R. Pacault 6 , C. Reppin 7 , \\ R. Rocchia ${ }^{8}$, A. Scheepmaker', H. Schnopper ${ }^{4}$, G. Spada 10, \\ R. Staubert ${ }^{11}$ and $M$. Turner ${ }^{12}$
}

'European Space Agency, SSD, ESTEC, Noordwijk, Netherlands ${ }^{2}$ Mullard Space Science Laboratory, University College, London,

U.K.

${ }^{3}$ Space Research Laboratory, Utrecht, Netherlands

-Danish Space Research Institute, Copenhagen, Denmark

SEuropean Space Agency, Rue Mario Nikis, Paris, France

'European Space Agency, FPSO, ESTEC, Noordwijk, Netherlands

'MPI für Extraterrestrische Physik, Garching, F.R.G.

'Section d'Astrophysique, Centre d'Etudes Nucléaires de Saclay,

France

${ }^{9}$ Cosmic Ray Working Group, Huygens Laboratory, Leiden,

${ }^{10}$ Istituto TESRE/CNR, Via de Castagnoli, Bologna, Italy

"Astronomisches Institut der Universitat Tübingen, F.R.G.

$12 X$-ray Astronomy Group, University of Leicester, U.K.

ABSTRACT

The salient features of $x-80$, a European $x$-ray Astrophysics Mission, and a candidate for selection as the next satellite in the European Space Agency's scientific programme, is described.

\section{INTRODUCTION AND SCIENTIFIC OBNECTIVES}

Through UHURU, Ariel $\mathrm{V}$ and HEAO-1, X-ray astronamy has been established as one of the most exciting and productive branches of astrophysics. Outstanding results have been recently obtained at longer wavelengths with the telescope of the Einstein Observatory and the European mission EXOSAT, to be undertaken in 1982 through 1984, is expected to make an extensive contribution in enlarging the scope of the subject.

Only a limited number of medium and high resolution spectroscopic observations up to $\sim 4 \mathrm{keV}$ could be carried out with Einstein. EXOSAT with its imaging telescopes will undertake medium and high resolution spectroscopy up to $\sim 2 \mathrm{keV}$, and with its medium energy experiment and gas scintillator experiment, medium resolution spectrosoopy up to $35 \mathrm{keV}$. However, a large programme of work on the detailed study of spectra and variability of $\mathrm{X}$-ray sources ( $(1 \mu \mathrm{JJy})$ can now be identified for a successor of the Einstein and EXOSAT observatories using new, sensitive instrumentation but which does not require the power and cost of large imaging telescopes.

X-rays are generated in high temperature $\left(T>10^{6} \mathrm{~K}\right.$ ) plasmas or by the interactions of highly energetic charged particles with magnetic fields or photons and are, in general, associated with sources and situations in the Universe which involve large concentrations of energy or where large energy releases are taking place.

High resolution spectroscopy in the energy range $0.5-10 \mathrm{keV}$ permits the study of the physics of energetic coronal and photoionised plasmas which are known to be present in many X-ray sources both inside and outside our galaxy. Measurements of emission line intensities in these plasmas will allow estimates of gas temperature, density and ionisation state, elemental abundance and gas velocity to be made for objects such as supernova remants, binary sources and clusters of galaxies.

Variability on all measured timescales is an almost universal characteristic of both galactic and extragalactic sources, and study of wich is a valuable tool for the investigation of their nature and emission processes. It ranges fran the sub-millisecond quasi-periodic bursts exemplified by Cygnus X-1 to the single cutbursts of bright galactic transient sources occupying many months, and possibly never recurring. In between there is a wide variety of behaviour, periodic and aperiodic at all timescales and intensities.

Spectral features above same $15 \mathrm{keV}$ have been discovered in a number of sources, e.g. Her $\mathrm{X}-1$. If the Her $x-1$ features are interpreted as cyclotron enission they may provide a powerful tool 
for the study of the magnetic fields of neutron stars. Many of the X-ray sources associated with active galaxies exhibit very hard spectra which suggests that much of the X-ray luminosity may be found in the energy range above $15 \mathrm{kev}$.

\section{SCIENTIFIC PAYLOAD}

The scientific aims of the mission require a combination of narrow field-of-view (Fov) instruments and wide-field instruments. The former allow the detailed study of spectral and temporal behaviour of selected, known sources, while the latter enable long term monitoring of galactic and extragalactic sources, as well as the positioning and temporal and spectral study of high latitude transients and bursters. The principal characteristics of the model payload and its variants studied in the ESA phase A study are given in Table 1. The instrument camplement includes four Bragg Crystal Spectrameters (BCS) and a Phoswich detector, together with either a Large Area Proportional Counter (IAPC) or a Kirkpatrick-Baez Concentrator (K-BC) or a Coded Mask Gas Scintillation Camera (OMGSC) as the narrow field instruments and four wide field-ofview Dicke or Transform Cameras (WFC). A small Gamma Ray Burst Monitor (GBM) is also included.

Table I $-X-80$ Payload Characterigtice

\begin{tabular}{|c|c|c|c|c|c|c|c|c|}
\hline & Instrument & $\begin{array}{l}\text { Energy/ } \\
\text { (Wavelength) } \\
\text { range }\end{array}$ & $\begin{array}{l}\text { Energy/ } \\
\text { (Wavelength) } \\
\text { resolution FWHA }\end{array}$ & $\begin{array}{l}\text { Sensitive } \\
\text { Area }\left(\mathrm{cm}^{2}\right)\end{array}$ & $\begin{array}{l}\text { Fov } \\
\text { (FWRZ) }\end{array}$ & $\begin{array}{l}\text { Angular } \\
\text { Resolution }\end{array}$ & $\begin{array}{l}\text { Source } \\
\text { Limits } \\
\text { Time(s) }\end{array}$ & $\begin{array}{l}\text { Confusion } \\
\qquad \text { Millicrabs }\end{array}$ \\
\hline . & $\begin{array}{l}\text { BCS } \\
\text { LiF(200) } \\
\text { PET1 } \\
\text { PET2 } \\
\text { TIAP }\end{array}$ & $\begin{array}{l}\text { Fe, } 1.7-2.0 \AA \\
\text { S: } 4.8-5.58 \\
\text { si, } 6.1-6.8 \& \\
0,18-22 \&\end{array}$ & $\begin{array}{l}2.3 \mathrm{mR} \\
4.0 \mathrm{mg} \\
6.1 \mathrm{~m} \\
92 \mathrm{mR}\end{array}$ & $\begin{array}{r}1440 \\
930 \\
695 \\
1470\end{array}$ & $6^{\circ}$ & $\therefore 1^{\prime}-5^{\prime}$ & & \\
\hline $2(a)$ & LAPC & $1.2-20 \mathrm{keV}$ & 197 at $6 \mathrm{keV}$ & 2500 & $1.5^{\circ}$ & - & $\approx 50$ & $\sim 0.3$ \\
\hline 2 (b) & $\mathrm{K}-\mathrm{BC}$ & $0.2-10 \mathrm{keV}$ & $\begin{array}{l}7 \pi \text { at } \mathrm{Fe} \\
9 \mathrm{at} \text { at } \\
12 \pi \text { at } \mathrm{Si} \\
20 \mathrm{x} \text { at } 0\end{array}$ & $\begin{array}{r}45 \\
400 \\
550 \\
700\end{array}$ & $30^{\prime}$ & $=41$ & $\approx 10^{4}$ & 20.06 \\
\hline 2(c) & $\operatorname{CMGSC}(2)$ & $2-30 \mathrm{keV}$ & 92 at $6 \mathrm{keV}$ & 700 & $3^{\circ}$ & $=5$ & $\sim 10^{4}$ & $\sim 0.06$ \\
\hline 3 & Phoswich & $15-200 \mathrm{keV}$ & $\begin{array}{l}30 z_{\text {: at }} 20 \mathrm{keV} \\
12 \pi \text { at } 200 \mathrm{keV}\end{array}$ & 650 & $4^{\circ}$ & - & & \\
\hline 4 & $\operatorname{WrC}(4)$ & $\begin{array}{l}2-20 \text { (imaging) } \\
2-50 \text { (timing) }\end{array}$ & 208 at $6 \mathrm{keV}$ & $\begin{array}{c}320 \\
\text { (each) }\end{array}$ & $34^{\circ}$ & $\approx 2^{\prime}$ & & \\
\hline 5 & GBM & $30-130 \mathrm{keV}$ & - & 50 & $120^{\circ}$ & $=10^{\circ}$ & & \\
\hline
\end{tabular}

The Bragg spectrameter offers good spectral resolution in four narrow energy bands centered on the strongest $\mathrm{X}$-ray lines emitted by highly ionised atans of the most abundant species (oxygen, silicon, sulphur and $i r o n)$, over a wide range of plasma temperatures from $\sim 10^{6} \mathrm{~K}$ to above $10^{8} \mathrm{~K}$. Spatially resolved spectral maps of extended sources can be produced with a resolution of a few minutes of arc.

The IAPC array has a large collecting area and, hence, capability for sub-millisecond timing on the bright galactic sources. The instrument has a low background and a narrow field-of-view, and can, therefore, study spectra and variability in faint extragalactic sources down to its confusion limit of 0.3 millicrabs on timescales of seconds to days. With its moderate energy resolution 1.2-20 keV, it complements the Bragg instrument and would provide continuum measurements over this range.

Advances in the development of light-weight technologies for minor manufacturing and the development of imaging gas scintillator cameras have lead to the study of the Kirkpatrick-Baez Concentrator and the coded Mask Gas Scintillation Camera as altematives to the LAPC in the $\mathrm{x}-80$ context. Since both instruments have imaging capability they can be used for spectral mapping. Their source confusion limits would be a factor of 5 lower than the IAPC. Since gas scintillation proportional counters are used in both instruments, the spectral resolution obtainable would be a factor of 2 to 3 better than the LAPC. Their lower area of course limits their capability for millisecond timing.

The WFCs will be able to image the X-ray sky with an accuracy of a few arc minutes from 2 to $20 \mathrm{keV}$ in order to detect, locate and measure X-ray transient events. They will provide a very efficient means of monitoring, on a regular basis, all bright $\mathrm{X}$-ray sources on timescales of days to years. Coordinated observations between $\mathrm{X}-80$ and ground observatories and indeod the Space Telescope should prove particularly fruitful. The WFCs can provide an alert to ground or other space facilities for transient-type phenomena.

The Phoswich will extend the range of the spectral and timing studies while the GBM, with its wide field of view, is intended to be capable of locating to a precision of about 10 arc minutes 25 or so high luminosity gamna-ray bursts expected per year. 


\section{SPACECRAFT AND MISSION PARAMETERS}

The mission can be readily undertaken on a free-flying satellite with modest performance requirements on 3-axis attitude control ( 5 arc min), attitude measurement ( 1 arc min) average data rate ( $40 \mathrm{kbps})$, power $(\sim 350 \mathrm{~W})$ and mass $(\sim 1$ tonne). The spacecraft would be launched by Ariane into a $600 \mathrm{~km}$ circular equatorial orbit, the best that can be envisaged from the background point of view, and operated for a minimum of 3 years. The spacecraft configuration is such that the Sun pointing face of the fixed solar array can be offset by up to $30^{\circ}$ fram the sun vector yet still be provided with adequate power. The spacecraft is free to roll about the solar vector so that the co-aligned, narrow-field-instruments, viewing perpendicular to the solar array normal, can view anywhere within a band on the sky, which is $60^{\circ}$ wide by $360^{\circ}$. The viewing band rotates around the sky with the Earth's motion and the whole celestial sphere is accessible within a six month season. Thus, any source can be observed by the narrow field instruments for up to 2 months at a time, every half year, and observing is then only intermupted by Earth occultation. The optical axes of the Wide Field Cameras are perpendicular to those of the narrow field instruments. The fields-of-view of the WFCs are $\sim 60^{\circ}$ wide and the four cameras are arranged such that with a roll about the solar vector and the use of the $30^{\circ}$ offset capability, a set of nine attitude manoeuvres is sufficient to allow an all-sky survey to be undertaken, excluding a cone of 300 half-angle centred on the Sun.

\section{X-80 PAYLOAD PERFOPMANCE CHARACTERISTICS AND OBSERVATION PROGRAMME}

The power of the $x-80$ instrumentation may be judged from the short sequence of diagrams. Figure 1 indicates the minimm detectable iron line strength (5 sigma) with the BCS, the K-BC and the CMGSC. Figure 2 indicates the minimum detectable source strength for the LAPC, WFC and OMGSC as a function of integration time.

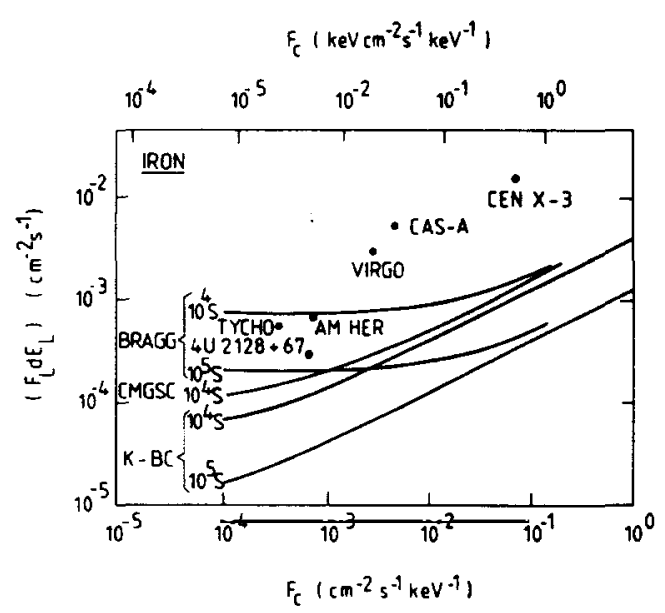

Fig. 1 Minimum detectable iron line strength (50) as.a function of source continuum strength at $6.7 \mathrm{keV}$. Sensitivities are shown as a function of observing time $\left(10^{4} \mathrm{~s}\right.$ and/or $\left.10^{5} \mathrm{~s}\right)$ for the Bragg Spectrometer, the Coded Mask Gas Scintillation Camera and the Kirkpatrick-Baez concentrator.

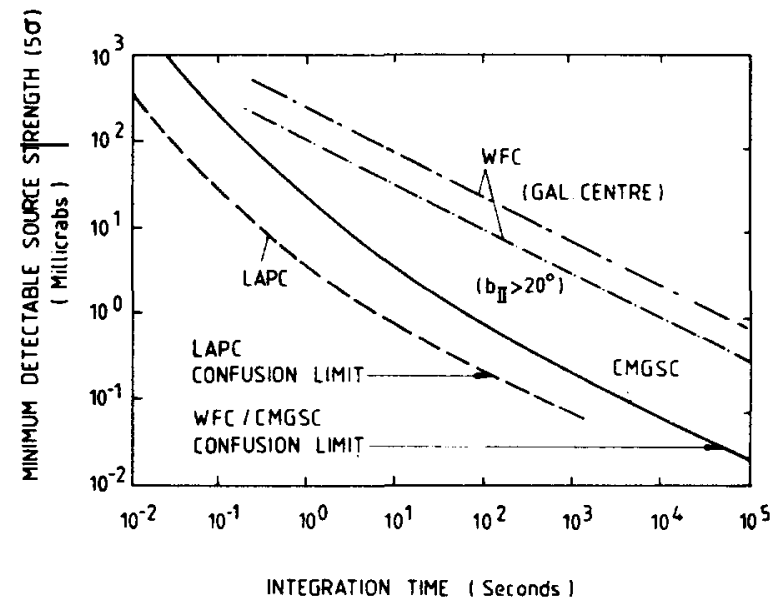

Fig. 2 Minimm detectable source strength (5\%) as a function of observation time for a Wide Field Camera, the Coded Mask Gas Scintillation Camera and the Large Area Proportional counter.

Figure 3 shows a simulation of an $\mathrm{X}-80$, WFC, observation of the galactic centre for $100 \mathrm{~s}$.

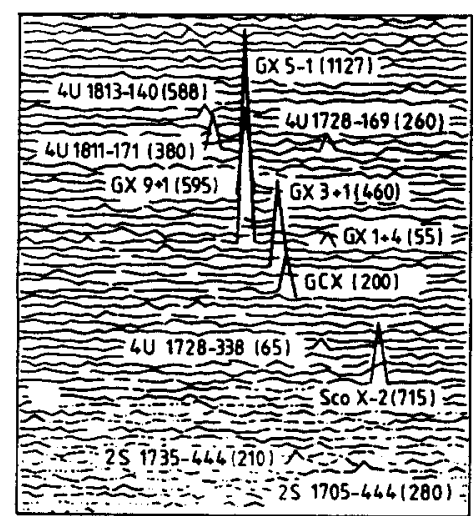

Fig. 3 Simulated performance of the Wide Field Camera for a $100 \mathrm{~s}$ observation of the galactic centre region. Incident source intensities (millicrabs) are shown in brackets. The WFC mask in this simulation has $64 \times 64$ elements yielding a $40^{\prime}$ resolution. The proposed instrument will have $512 \times 512$ elements yielding $\sim 5^{\prime}$ resolution. 
Figure 4 shows a simulation of the residual emission line spectrum of CAS-A as observed with the $\mathrm{K}-\mathrm{BC}$. The sensitivity of the WFC to intensity changes is illustrated in Figure 5 . Figure 6 shows the sensitivity of the Phoswich detector.

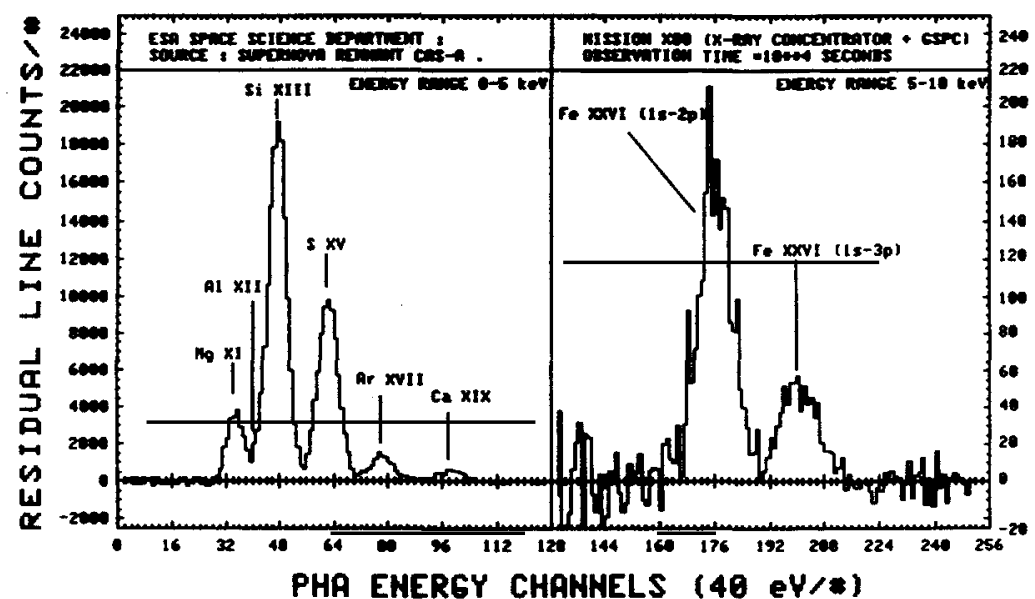

Fig. 4 Simulation of the residual emission line spectrum developed by the Kirkpatrick-Baez Concentrator and gas scintillation focal plane detector for a $10^{4} \mathrm{~s}$ observation of the CAS-A SNR

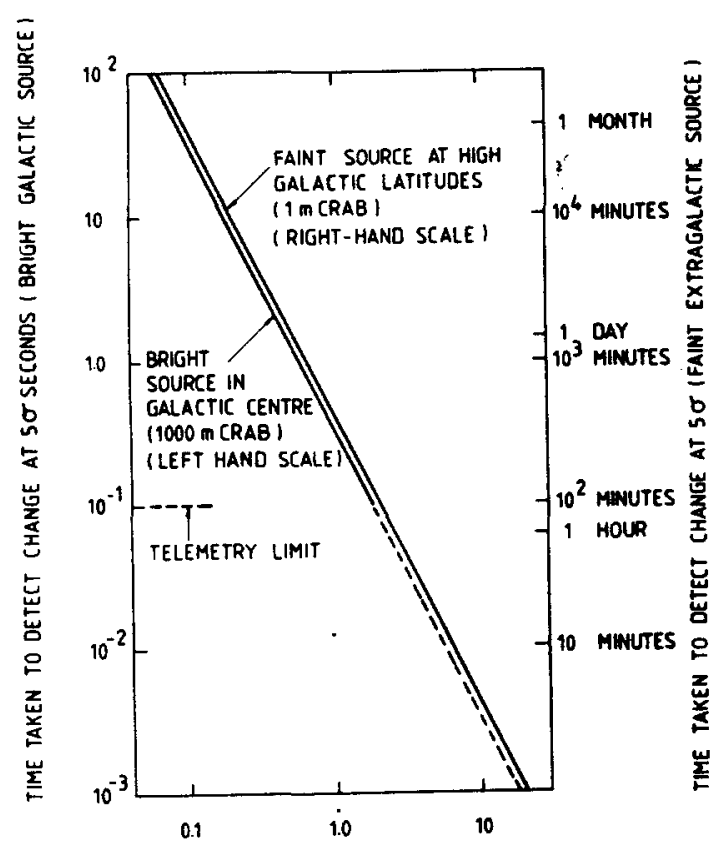

FRACTIONAL CHANGE IN SOURCE INTENSTTY

Fig. 5 Sensitivity of the wide Field Camera to changes in source intensity for a bright source $\left(10^{3}\right.$ millicrab) in the galactic centre region and a faint source (1 millicrab) at high galactic latitudes.

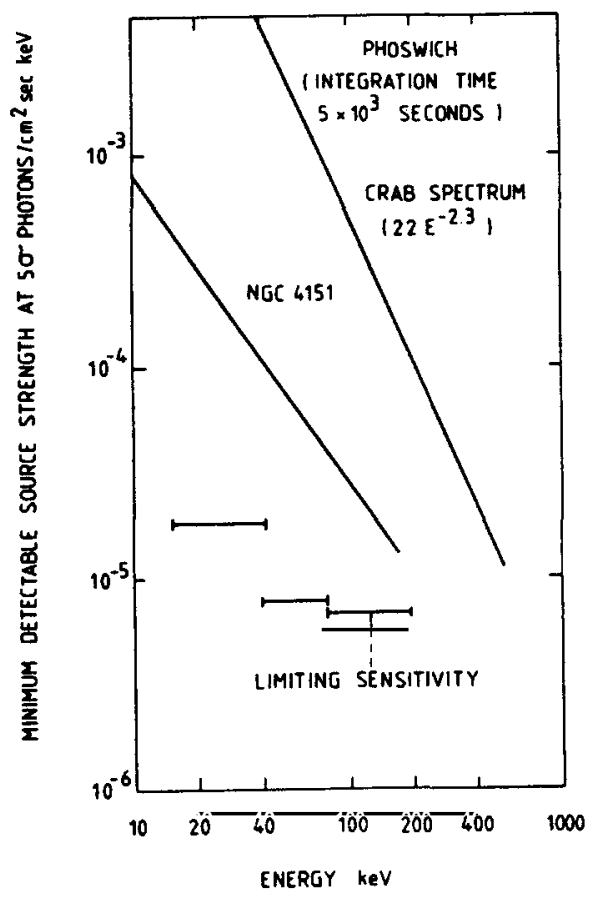

Fig. 6 Minimum detectable source strength as a function of energy for a $5 \times 10^{3}$ second observation with the Phoswich detector. The sensitivity with respect to the crab and NGC 4151 is indicated. 
Table II indicates in a qualitative manner the relevance of the proposed instruments to the different source types listed. Generally speaking targets will be examined by the narrow field instruments for $10^{4}-10^{5}$ seconds. On the other hand the minimu observation time will be of the order $10^{3}$ seconds, since the spacecraft will not adopt more than one pointing attitude per orbit. A camplete sky survey to the $1 \mu J y$ level with the WFCs will require same 3 days.

Table III outlines an observing programme for a three year mission. It should be borne in mind that simultaneous abservations are possible, on different fields, with the narrow- and wide-field instruments. Given selection of the mission early in 1983, the satellite could be launched in 1987, and operated to at least 1990. While it is anticipated that the instruments would be of the principal investigator type, it is intended that a significant guest observer prograrme would be implemented.

Table II. Compatibility of the Proposed Payload

\begin{tabular}{|c|c|c|c|c|}
\hline Source Type & Bragg & $\begin{array}{l}\text { IAPC, } \\
\mathrm{K}-\mathrm{BC} \text {, } \\
\text { GXSC }\end{array}$ & Fhoswich & $\begin{array}{c}\text { Wide } \\
\text { Field } \\
\text { Cameraso }\end{array}$ \\
\hline $\begin{array}{l}\text { osox } \\
\text { Active galaxy } \\
\text { Cluster } \\
\text { Binary } \\
\text { Burster } \\
\text { Transient } \\
\text { SNR } \\
\text { Active starx } \\
\text { Stellar corona } \\
\text { Bulge soumce }\end{array}$ & 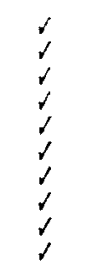 & 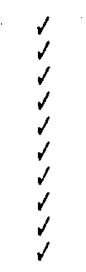 & 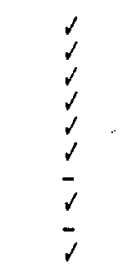 & 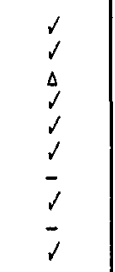 \\
\hline
\end{tabular}

Table III. $x-80$, Model Three Year Mission Observing Progranme

\begin{tabular}{|lr|}
\hline 1. 50 QSO's and active galaxies & 400 days \\
2. 50 clusters of galaxies & 200 days \\
3. 50 X-ray binaries & 200 days \\
4. 50 active stars and coronae & 100 days \\
5. 20 supernova remants & 50 days \\
6. Sky survey with WFC & 300 days \\
7. Galactic centre region with WFC) & 50 days \\
\hline
\end{tabular}

$x_{\text {Simultaneous ground based observation worthwhile }}$ $\triangle_{W F C}$ can monitor individual galaxies in cluster owrC can provide alert to other observatories

\section{ACKNOWLEDGEMENTS}

The authors, members of the ESA X-80 Phase A Study Team, wish to acknowledge the advice frcm and many useful and stimulating discussions with Drs. I. Culhane, L. Koch, A. Peacock and $\mathrm{K}$. Pounds. 